Print- ISSN: 1391-586X, E- ISSN: 2602-9030, Copyright @ 2017 by Faculty of Science, Eastern University,

Sri Lanka.

\title{
LINEAR PROGRAMMING APPROACH FOR DOCTORS SCHEDULING IN A HOSPITAL
}

\author{
Paramadevan, $\mathrm{P}^{1}$ \\ ${ }^{1}$ Department of Mathematics, Faculty of Science, Eastern University, Sri Lanka
}

\begin{abstract}
The main objective of this study is to propose an optimization model for achieving efficient scheduling of doctors attached to any hospital. In the recent literatures, a wide variety of different methodologies and models have been developed pertaining to various scheduling problems. These include Mathematical Programming, MetaHeuristic Methods and Constraint Satisfaction Techniques. First, an optimization model is formed by formulating the objectives and the constraints of the problem mathematically. Further, the real doctors' scheduling problem is formed as a 0-1 Integer Linear Programming problem. To solve the problem, Branch-and-Bound technique is applied by using the optimization software package LINGO. Finally, the solution to the optimization problem is converted to a regular doctors' schedule. The methodology is illustrated by preparing a weekly schedule for a private hospital in Sri Lanka which has 32 doctors of the same grade.
\end{abstract}

Keywords: Doctor scheduling, Mathematical Programming, Meta Heuristic Methods, Constraint Satisfaction Techniques, Column generation, Branch-and-Bound technique.

${ }^{1}$ Corresponding author: paramdeve@yahoo.com.au

\section{INTRODUCTION}

Sri Lanka holds a unique position in South Asia as one of the less developed nations to provide universal health at almost free of charge. Sri Lankan government health service institutions and public hospitals have huge percentage of patients visiting every day for treatment and consultation compare to private hospitals. 
In Sri Lankan hospitals, doctors try to provide tremendous service for public to solve their health-related issues in time. However, it is observed that many doctors are dissatisfied with their jobs, which is mainly due to their long working hours, and continuous night schedules without proper rest. This can affect patient care and reduce quality of the service. The main issue is that the current doctors scheduling systems in place are not intelligent enough to bring a unique scheduling solution satisfying various demands without affecting even the health and personal preferences of doctors. One of the possible mechanism to reduce the stress of the doctors may be that, prepare roster with utmost satisfaction of their preference on working day or shifts. Hence, the major objective of this study is to create a mathematical model related to the real-world problem arising from the operations of a hospital, which termed as Doctors Scheduling Problem (DSP) and propose a method to find solution in an efficient way.

A doctor schedule provides an assignment to doctors who need to perform different duties in the roster. Unlike Nurse Scheduling Problems (NSP) [1], in doctors scheduling, maximizing satisfaction only matters, since authors' retention is the most critical issue faced by hospital administrators in most of the western countries [2]. In addition, while nurse schedules must adhere to collective union agreements, doctor's schedules are more driven by personal preferences. In general, the DSP need to consider many conflicting constraints along with various preferences from doctors. In [2], the authors provide the fundamental difference between DSP and NSP. According to available literature DSP focuses primarily on a single type of duty, such as the emergency treatment unit [3], the operation theatre [4], and the physiotherapy unit [5].

This study proposes a mechanism based on 0-1 integer linear programming to prepare a roster for a group of doctors attached to a hospital for a period of one week. The proposed method is less complex compared to the existing methods since the approach Branch-and-Bound is a common technique in the scheduling field. Further, this approach does not only provide an idea to prepare a roster but it also allows the inclusion of some additional features which is more challenging in the manual roster preparation. The remainder of the paper is organized as follows. Section II, describes in detail the proposed optimization model for doctors scheduling. Section III presents the results obtained by applying our optimization model to a hospital in Sri Lanka. Section IV discusses the results and draw the conclusions. 


\section{THE PROPOSED MODEL}

To presenting the optimization model, the notations and meanings of technical terms that are being used in the sequel is explained in this section.

1. Let $C=\{1,2, \ldots \ldots . \bar{c}\}$ be the set of doctors. For any $c \in C, Y_{c}$ and $Z_{c}$ be the maximum number of day shifts and maximum number of night shifts to be assigned for the doctor $c$ within the scheduling period respectively.

2. Let $W=\{1,2, \ldots \ldots . \bar{w}\}$ be the set of wards/units in the hospital to which doctors to be assigned.

3. Let $T=\{1$ (morning), 2(evening),3(noon), 4 (night) $\}$

be the set of shift types. Here, all the shift types 1,2,3 and 4 have equal length of 6 hours starting from morning $6 \mathrm{AM}$ onwards. In this paper shift types 1,2 and 3 are mentioned as day shifts while shift type 4 is mentioned as night shift, it starts from mid night 12.00 in the particular day and ends 6 AM in the next day.

4. Let $D=\{1,2, \ldots \ldots . \hat{\phi}\}$ be the days in the scheduling time horizon.

5. Let $R_{w_{-} d_{-} t}$ to be a demand for number of doctors to be at the ward $w \in W$ on a day $d \in D$ of shift type $t \in T$.

6. Let $k_{1}$ be the maximum number of consecutive day shifts, while $k_{2}$ be the maximum number of consecutive night shifts within the scheduling period respectively.

7. Let $P_{c_{-}} w_{-} d_{-} t$ to be a penalty occurs, if the doctor $c \in C$ is scheduled to work at the ward $w \in W$ shift type $t \in T$ on a day $d \in D$.

Here the required task is to assign doctors for different shift types to different wards according to the demand on each shift on a day in the planning time horizon. It should satisfy the following standard requirements which are common to most of the DSP.

a) Demand at any shift type on a particular day should be satisfied.

b) A doctor should be assigned for at most one shift among four available shifts on a day. 
c) At most maximum number of working days/day-shift should be assigned for a doctor within the time horizon considered.

d) At least minimum number of day shifts should be assigned for a doctor within the time horizon.

e) At most maximum number of night shifts should be assigned for a doctor in the time horizon.

f) At least minimum number of night shifts should be assigned for a doctor in the time horizon.

In addition to these standard requirements following special requirements are request by the doctors:

g) Shift type 1 should not be assigned for a doctor if he/she assigned for a night shift (shift type 4) of the previous day.

h) Maximum number of consecutive working days is 3 .

i) Maximum number of consecutive night shifts is 2 .

Next, the decision variables are being introduced. Let $x_{n_{-} w_{-} d_{-} t}=\left\{\begin{array}{l}1, \text { if doctor } n \text { is assigned to the ward } w \text { for the shif type ton } a \text { day } d \\ 0, \text { other wise. }\end{array}\right.$

The optimization model which incorporates both common and special requirements can be presented as:

\section{Minimize}

$$
\sum_{n \in N} \sum_{w \in W} \sum_{d \in D} \sum_{t \in T} p_{c_{-}} w_{-} d_{-} t^{x_{C}-w_{-} d_{-} t}
$$

Subject to

$$
\begin{aligned}
& \sum_{c \in C} x_{c_{-}} w_{-} d_{-} t=R_{w_{-}} d_{-} t, \forall w \in W, d \in D, t \in T \\
& \sum_{w \in W} \sum_{t \in T} x_{C_{-}} w_{-} d_{-} t \leq 1, \quad \forall c \in C, d \in D \\
& \sum_{w \in W} \sum_{d \in D} \sum_{t \in T} x_{c_{-} w_{-} d_{-} t} \leq Y_{c}, \quad \forall c \in C
\end{aligned}
$$




$$
\begin{aligned}
& \sum_{w \in W} \sum_{d \in D} \sum_{t \in T} x_{c} w_{-} d_{-} t \geq y_{c}, \forall c \in C \\
& \sum_{w \in W} \sum_{d \in D} x_{c_{-}} w_{-} d_{-} 4 \leq Z_{c}, \forall c \in C \\
& \sum_{w \in W} \sum_{d \in D} x_{c_{-}} w_{-} d_{-} 4 \geq z_{n}, \forall c \in C \\
& x_{c_{-} w_{-} d-1_{-}{ }^{4}}+\sum_{w \in W} x_{c_{-} w_{-} d_{-1}} \leq 1, \forall c \in C, w \in W, d \in D \\
& \sum_{w \in W} \sum_{d=l}^{k_{1}+l} x_{c_{-} w_{-} d_{-} t} \leq k_{1}, \forall c \in C, l \in\left\{1,2, . ., Y_{c}\right\} \\
& \sum_{w \in W} \sum_{d=l}^{k_{2}+l} x_{c_{-} w_{-} d_{-} t} \leq k_{2}, \forall c \in C, l \in\left\{1,2, . ., Z_{c}\right\}
\end{aligned}
$$

The mathematical model described above is for preparing a work schedule for doctors in a hospital. These schedules must respect working contracts and meet the demand for a given number of doctors on a ward in each shift type, while being perceived to be fair by the doctors themselves. The objective function of our optimization model is considered as a cost function, where cost is interpreted as penalty and penalty is defined based on the undesirability of a doctor to work at a particular ward at a particular shift type on a particular day. Therefore, our attempt is to minimize the penalty subject to the given constraints. These penalties appeared as coefficients in the objective function.

\section{EVALUATION, RESULTS AND DISCUSSION}

The hospital that considered in this paper as a practical example for applying this mathematical model consists 32 doctors and 4 wards. The hospital administration requires only one doctor for the ward number 1 and 2 for ward numbers 2, 3 and 4 at each shift type in a day. Hence, the demands considered in the mathematical model $R_{1} d_{-} t$ is equal to 1 while $R_{2} d_{-} t, R_{3} d_{-} t$ and $R_{4_{-} d_{-} t}$ are uniform and is equal to 2 for all shifts throughout the time horizon. Also, the maximum number of working days and maximum number of night shifts for a doctor are required to be 5 and 3 respectively. That is, $Y_{C}=5$ and $Z_{C}=3$ while minimum number of working days and 
minimum number of night shifts are required to be 4 and 1 respectively. Further, the maximum number of consecutive working days, $k_{1}$ and maximum number of consecutive night shifts, $k_{2}$ within the scheduling period are required to be 3 and 2 respectively.

By using the above information obtained from the hospital administration as well as the doctors attached. A 0-1 integer linear programming model is formulated, which consists of 3584, 0-1 binary decision variables and 1553 constraints. The result obtained by solving the 0-1 Integer Linear Programming problem using the LINGO software package is presented in the table 1 . Here, numbers represent the shift type for corresponding days:

In this table first row, 'Day' represents day number and the first column 'Ward' represents ward number. Since the demand for the doctors in the ward number 1 is 1 , the doctor number (D.No) 28, 19, 17, 29 are assigned for the available four shifts (Shift.No) respectively on the day 1 . While demands are 2 for all other three wards, two doctors are assigned for each shift. For example, doctors with number 19 and 13 have duty in the shift number 1, 14, and 25 have in the shift 2, 4 and 21 have in the shift no 3 and 2,29 have night duty in the ward number 2 on day number 1 . From the above table, it can be observed that this hospital runs with shortage of doctors and the doctors are working beyond the limit, when considered the number of working days. Eight of them working the whole 7 days while some working 6 days. Only 5 of them are working for the acceptable number of working days that is 5 . 
Table 1: Doctors' schedule

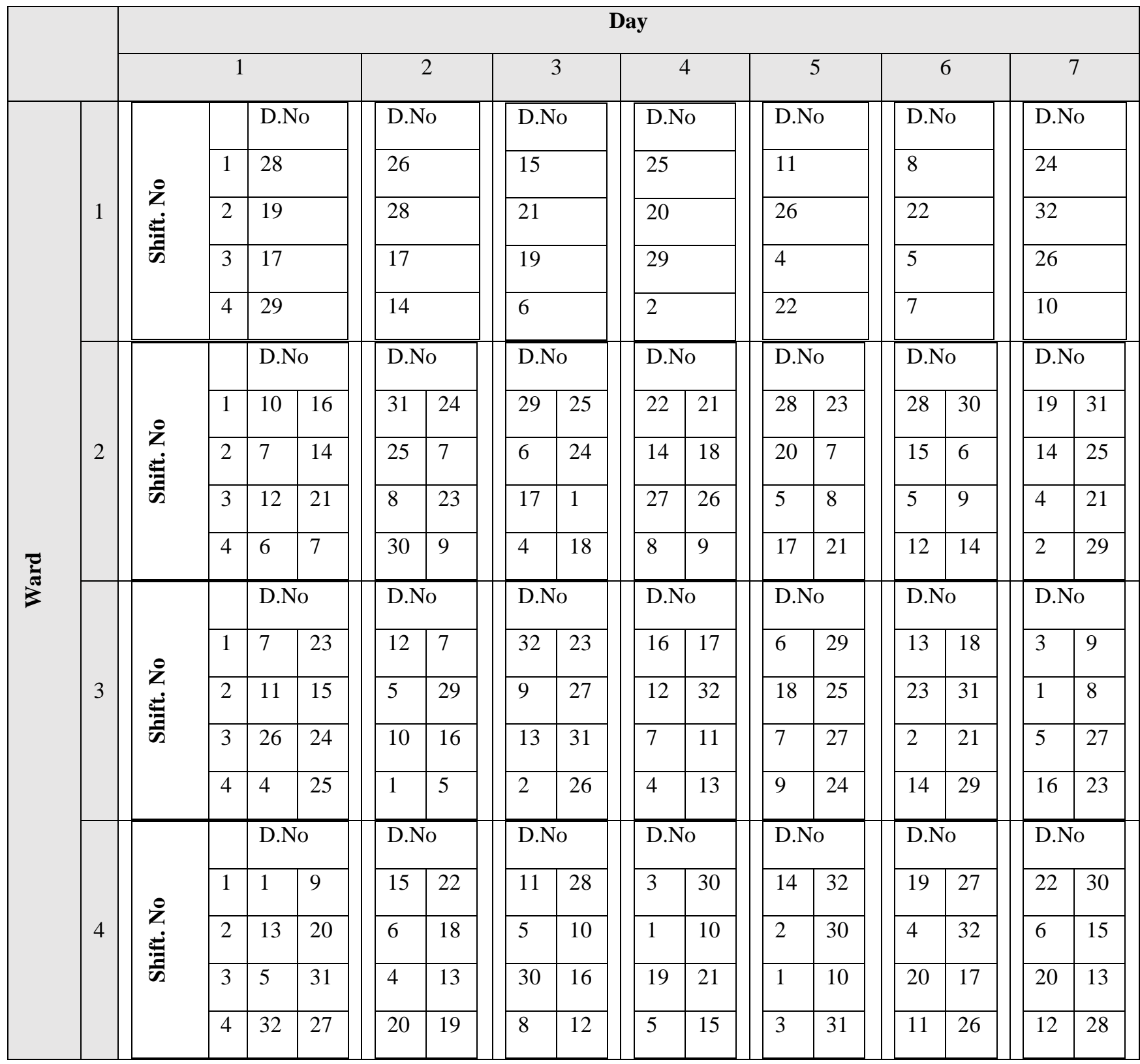

D.N: Doctor Number 


\section{CONCLUSION}

An optimization model for doctors scheduling has been developed. Also a method of solution to solve the model has been proposed. This method will guide to prepare a doctor schedule more efficiently, accurately and quickly for any hospital which consists of any number of wards which satisfies the doctors' preferences and regulations provided by the hospital administrators. This method also helps the administration to study the overload situations where it requires an appointment of new doctors. In our model, all doctors are considered as same grade. In reality doctors are in different grades such as Consultant, Senior Registrar, Registrar, House Officer etc. And their demands also vary according to their qualifications/experience. One of our future works in this study will incorporate the grades in the formulation of the mathematical model and this will produce more realistic results.

\section{REFERENCES}

[1] Paramadevan, P., and Daundasekera, W.B., (2013). Nurse scheduling based on Branch-and-Bound algorithm. International journal of Scientific and Research Publications, 3(7):1-7.

[2] Carter, M.W., and Lapierre, S.D., (2001). Scheduling emergency room physicians. Health Care Management Science, 4:347-360.

[3] Beaulieu, H., Ferland, J.A., Gendron, B., and Philippe, M., (2003). A Mathematical programming approach for scheduling physicians in the emergency room, Health Care Management Science, 3:193-200.

[4] Burke, E.K., and Riise, A., (2008). A Surgery allocation and scheduling. In proceedings of the $7^{\text {th }}$ International Conference of Practice and Theory of Automated Timetabling, Montreal, Canada, 18-22.

[5] Ogulata, S.N., Koyuncu, M., .and Karakas, E., (2008). Personnel and patient scheduling in the high demanded hospital services: a case study in the physiotherapy service, Journal of Medical Systems, 32(3):221-228. 